\title{
Articulo Original / Original Article \\ Ethnobotany as a parameter for the study of cultural mimicry among Roma people
}

\author{
[La etnobotánica como parámetro para el estudio del mimetismo cultural entre el pueblo gitano]
}

\author{
Ricardo Alexandre de Araújo Monteiro Lobo, Ana Cristina Bastos Nigro Monteiro Lobo, \\ Antônio Fernando Morais de Oliveira \& Laise de Holanda Cavalcanti Andrade
}

Department of Botany, Federal University of Pernambuco, Recife, Brazil.

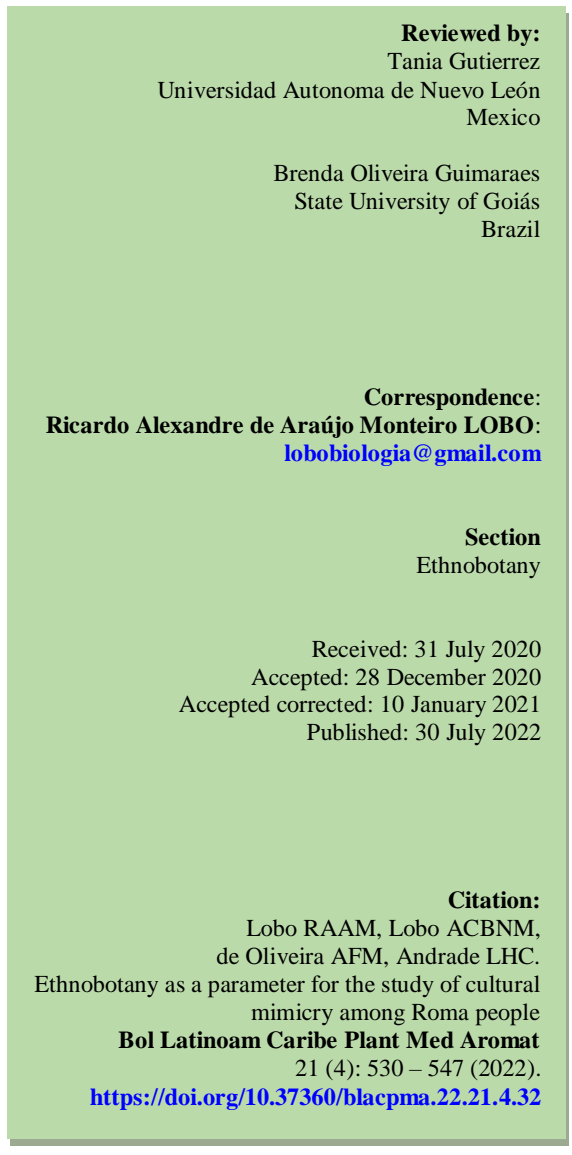

Abstract: The nomadic behavior of Roma people has allowed their cultural interaction with people from different continents. Brazil has received Roma immigrants since its colonization, and there are currently more than 800,000 Roma people among the Brazilian population. This article describes the ethnobotanical knowledge of two Calon families (Alves and Dantas) established in hinterland cities of Pernambuco, northeastern Brazil. The survey was conducted with 23 informants $(\geq 40$ years old, $85 \%$ of the target audience) selected through the snowball technique and using semi-structured interviews. The plants mentioned were collected, identified and deposited in the IPA and UFP herbaria. The 157 species (85 native) are used for food, condiment, cosmetic, medicinal, ritualistic, technological, veterinary, and other purposes. The useful flora and forms of use are similar to those reported by non-Roma communities living in the same region, showing a kind of mimicry adopted by the Roma people, yet maintaining their cultural identity.

Keywords: Medicinal plants; Useful plants; Calon ethnicity; Traditional communities.

Resumen: El comportamiento nómada de los gitanos ha permitido su interacción cultural con personas de diferentes continentes. Brasil ha recibido inmigrantes romaníes desde su colonización y actualmente hay más de 800.000 romaníes entre la población brasileña. Este artículo describe el conocimiento etnobotánico de dos familias Calon (Alves y Dantas) establecidas en ciudades del interior de Pernambuco, noreste de Brasil. La encuesta se realizó con 23 informantes ( $\geq 40$ años, $85 \%$ del público objetivo) seleccionados mediante la técnica de bola de nieve y mediante entrevistas semiestructuradas. Las plantas mencionadas fueron recolectadas, identificadas y depositadas en los herbarios IPA y UFP. Las 157 especies (85 nativas) se utilizan para alimentos, condimentos, cosméticos, medicinales, ritualistas, tecnológicos, veterinarios y otros fines. La flora útil y las formas de uso son similares a las reportadas por las comunidades no gitanas que viven en la misma región, mostrando una especie de mimetismo adoptado por el pueblo gitano, pero manteniendo su identidad cultural.

Palabras clave: Plantas medicinales; Plantas útiles; Etnia Calon; Comunidades tradicionales. 


\section{INTRODUCTION}

Scattered throughout the world, Roma people are a non-territorial nation characterized by a strong cultural identity which still has much to reveal (Andrade Júnior, 2013). Linguistic and genetic research shows Romanies originated in the northwest region of present-day India, from where they dispersed from the $10^{\text {th }}$ century onwards (Gresham et al., 2001; Mendizabal et al., 2012). They arrived in Europe in around the $14^{\text {th }}$ century, settling in a region of Greece known at the time as Little Egypt; the names by which they are known - gypsies (English), gitanos (Spanish) and ciganos (Portuguese) - are derived from the mistaken historical belief that they originated in this region (Moonen, 2011).

The nomadic way of living of Roma people has led them to incorporate part of the culture of nonRoma people with whom they have interacted throughout their history. Examples of this adaptation are perceived in the chib language, which incorporated words from other languages throughout its evolution, as well as the songs and dances of the Gadje (non-Roma people) and the clothes and religions of the communities through which Roma people passed (Ramanush, 2011; Simões, 2014). The same likely happened with the diverse Romanies' uses of flora and fauna, which were possibly incorporated from other cultures and merged with their own traditions.

The incorporation of a culture is a mimetic process that involves not only material products but also forms of action, social relations and representations. The readiness for mimicry is fundamental for learning and for cultural transmission, allowing the development and transformation of a given culture (Wulf, 2016). The use of local flora is likely a parameter yet to be considered in studies of the cultural mimicry experienced by Roma people over time, constituting an aspect yet to be explored and clarified.

Romanies began to arrive in Brazil in the $16^{\text {th }}$ century, dispersed throughout all regions of the country over the following centuries and, since 2006, became part of the traditional communities of Brazil (Coelho, 1892; Teixeira, 2008; Moonen, 2011). According to data from the Brazilian Institute of Geography and Statistics (IBGE, 2011), there are about 800,000 Roma people in the country, with 291 registered camps distributed in 21 Brazilian states.

Several forms of use of plant resources are part of the Roma culture, as has been reported for communities living in European and Asian countries such as France and Bangladesh (Derlon, 1979; Seraj et al., 2013). Ethnobotanical research on the relations of Roma communities established in Brazil with the local flora was initiated by Lobo et al. (2020), who reported the use of 10 species for the treatment of domestic animals. Continuing this line of research, the present study inventoried the plants used by Roma people of the Calon ethnic group settled in Pernambuco, northeastern Brazil, and compared the list of species and their respective uses with those provided in surveys of non-Roma communities that inhabit the Agreste and humid forest zones, aiming to identify cultural similarities and differences.

\section{MATERIALS AND METHODS}

The research was carried out with authorization of the Genetic Heritage Management Council (CGen) of the Ministry of the Environment (registration A9CB221), and the Ethics Council of the Federal University of Pernambuco (process 57999516.2.0000.5208). The president of the Association of Roma in Pernambuco (ACIPE), Mr. Enildo Kalon, mediated the first contacts introducing the heads of the Dantas family residing in the municipality of Itambé, Atlantic forest Mesoregion - and Alves family - residing in the municipalities of Altinho, Caruaru and Feira Nova, in the Agreste Mesoregion (Figure No. 1). The region where these municipalities are located has low-flow perennial rivers and low underground water potential. Average annual temperatures vary between $24^{\circ} \mathrm{C}$ and $27^{\circ} \mathrm{C}$ and the average annual rainfall is $1147.2 \mathrm{~mm}$. The vegetation is formed by deciduous and subdeciduous forests and the climate is tropical rainy, with a dry summer and a rainy season that starts in January/February and ends in September (SGM MME, 2005).

Informed Consent Forms were presented to the heads of the two families and, after participating in the semi-structured interviews, members of the respective families aged $\geq 40$ years were selected through the "snowball" technique to participate as informants (Albuquerque et al., 2010). The interviews were conducted individually with 11 men and 12 women, corresponding to $85.2 \%$ of the target audience, in 26 visits to the communities. The set of informants from the two Roma families was analyzed according to gender and age group, the latter being divided into three classes: 40 to 50 years, 51 to 60 
years, and over 60 years.

Figure No. 1

Municipalities of Pernambuco, northeastern Brazil, where the Roma families were interviewed. Maps of Brazil and Pernambuco state were obtained and adapted from Wikipedia Commons (Wikimedia Foundation, Inc., San Francisco, California, USA)

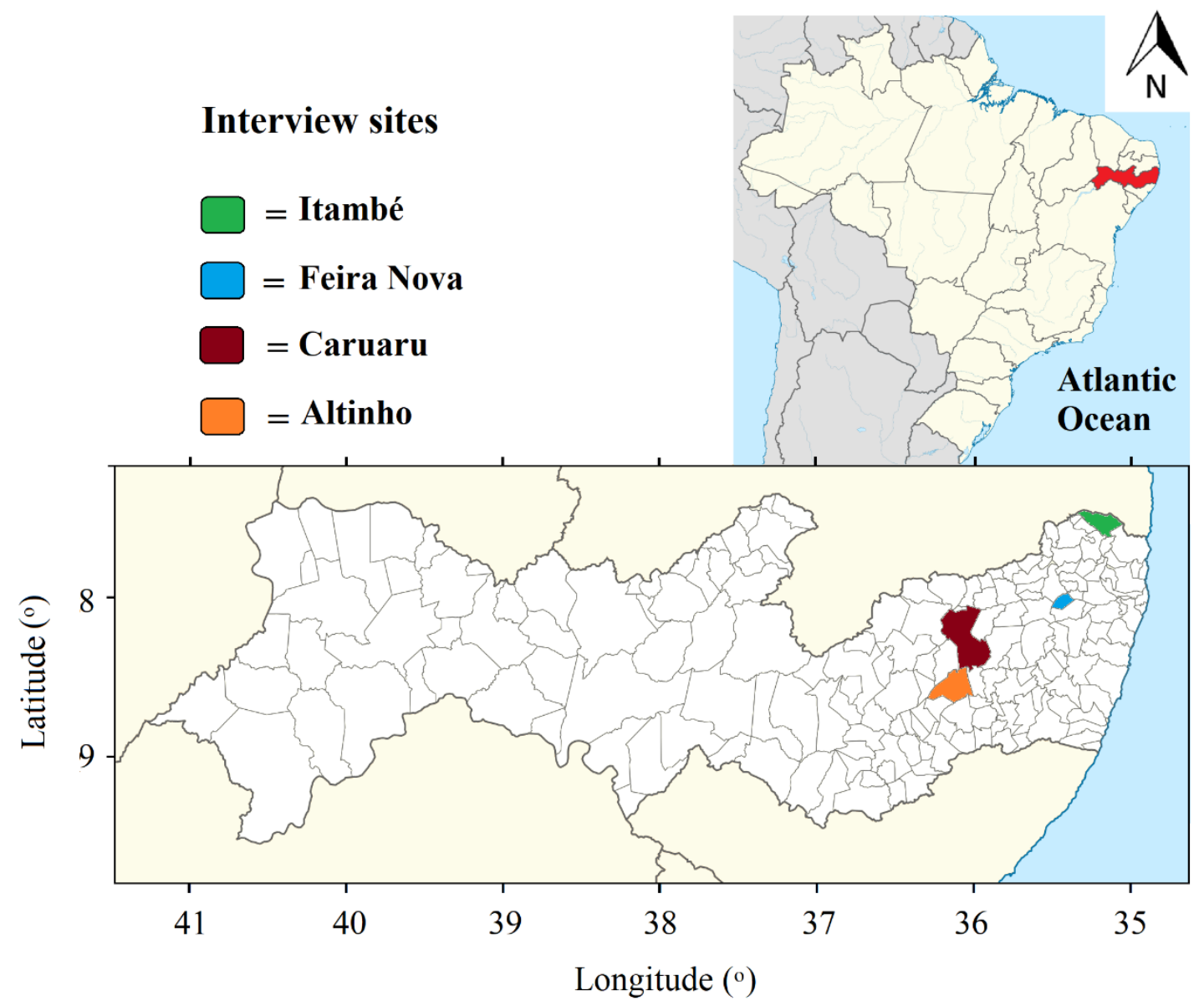

In addition to the interviews, guided tours were held with the informants for observation and collection of the plants mentioned in gardens, backyards, vacant lots, streets and farms. After being identified by taxonomists from the Agronomic Institute of Pernambuco, representative exsiccates of the mentioned species were registered in the IPA and UFP herbaria. The species were classified into native or exotic (naturalized or cultivated) according to data from Flora do Brasil (2020).

After the survey, the mentioned species were classified into the following categories of use: food, condiment, cosmetic, medicinal, ritualistic, technological, veterinary, and others (Prance et al., 1987). The International Statistical Classification of Diseases and Related Health Problems (ICD) was used to relate diseases, symptoms, signs, abnormalities, complaints and external causes of injuries treated by plants cited by the informants with the scientific nomenclature. The following codes were used: A06: Amoebiasis; A37.9: Whooping cough; B05: Measles; B07: Viral warts; B36.0: Pityriasis versicolor; B65.1: Schistosomiasis due to Schistosoma mansoni; B82.0: Intestinal

Boletín Latinoamericano y del Caribe de Plantas Medicinales y Aromáticas / 532 
helminthiasis; C80: Malignant neoplasm, without specification of site; D53.9: Anemia and stomach pain; E14: Diabetes mellitus; E65: Localized adiposity; E78.0: Hypercholesterolemia; F50.9: Eating disorder; F51.0: Insomnia; H10: Conjunctivitis; H25: Senile cataract; H66.9: Otitis media; H83.0: Labyrinthitis; I10: Essential hypertension; I64: Stroke, unspecified; I74: Arterial embolism and thrombosis; I84: Hemorrhoids; J01: Acute sinusitis; J02: Acute pharyngitis; J11: Influenza, virus not identified (flu); J40: Bronchitis, not specified as acute or chronic; J45: Asthma; K05: Gingivitis and periodontal diseases; K08.9: Disorder of teeth and supporting structures; K25: Gastric ulcer; K29.7: Gastritis; K30: Functional dyspepsia; K59.0: Constipation; K77.0: Liver disorders in infectious and parasitic diseases; L21: Seborrhoeic dermatitis; L30.9: Dermatitis, unspecified; L65.9: Nonscarring hair loss; L80: Vitiligo; L98.9: Disorder of skin and subcutaneous tissue; M25.5: Pain in joint; M54.6: Pain in thoracic spine; N20.9: Urinary calculus; N39.0: Urinary tract infection, site not specified; N41: Inflammatory diseases of prostate; N71.9: Inflammatory disease of uterus; N76.0: Acute vaginitis; N94: Pain and other conditions associated with female genital organs and menstrual cycle; O06: Unspecified abortion; R05: Cough; R10: Abdominal and pelvic pain; R11: Nausea and vomiting; R33: Retention of urine; R42: Dizziness and giddiness; R45.0: Nervousness; R50.9: Fever, unspecified; R51: Headache; R52.0: Acute pain; R53: Malaise and fatigue; R60.0: Localized oedema; T14.1: Open wound of unspecified body region.

The International Classification of Functioning, Disability and Health (ICF) established by the World Health Organization (WHO) was used to calculate the reliability index of the 10 medicinal species that were cited by one third or more of the informants, according to the formula:

$$
I_{\text {Fid }}=\left(\frac{N I F}{N I T}\right) \times 100 \%
$$

where NIF = number of informants who cited the most common functioning for the species; NIT = number of informants who cited any functioning for the species (Martins \& Costa, 2016).

The Relative Importance (RI) was calculated for the 10 most cited medicinal species, based on
Bennett \& Prance (2000), according to the formula: $\mathrm{RI}=\mathrm{NBS}+\mathrm{NP}$, where NBS = number of body systems influenced by the cited species, calculated as the ratio between the number of body systems treated by the cited species (NBSS) and the total number of body systems treated by the most versatile species (NBSVS); and NP = number of properties, calculated as the ratio between the number of properties attributed to a given species (NPS) and the total number of properties attributed to the most versatile species.

The use value (UV) of each species was calculated as the ratio between the sum of the number of uses mentioned $(\Sigma U)$ and the total number of respondents in the survey (n), as shown in the formula: $\mathrm{VU}=\left(\frac{\Sigma U}{n}\right)$ (Albuquerque et al., 2010).

The Sørensen index was used to compare the similarity between the sets of plants cited as useful, calculated by the formula: $\frac{2 c}{a+b}$, where"a" represents the number of species mentioned by the Dantas family,"b" the number of species cited by the Alves family, and"c"the number of species cited by both families (Silva \& Andrade, 2005).

\section{RESULTS}

One hundred and fifty seven (157) plant species were cited as useful. They were distributed in 64 families of Angiosperms (Table No. 1). Fabaceae (19 spp.), Lamiaceae (11 spp.), Euphorbiaceae (9 spp.), Cucurbitaceae (7 $\begin{array}{llll}7 & \text { spp.), Solanaceae }(7 & \text { spp.), }\end{array}$ Malvaceae (6 spp.), Myrtaceae (6 spp.), and Rubiaceae (5 spp.) stood out in number of species cited. Aloe vera ("babosa"), Anacardium occidentale (cashew), Citrus spp. (orange), Cocos nucifera (coconut tree), Cymbopogon citratus (lemon grass), Lippia alba ("erva cidreira"), Mentha sp. (small leaf mint), Musa spp. (banana), Myracrodruon urundeuva ("aroeira") and Punica granata (pomegranate) were the most mentioned species, with 12 to 24 citations each. The highest use values $(0.26-0.22)$ were recorded for $A$. occidentale, $C$. citratus, $C$. nucifera, Coriandrum sativum, L. alba, Mentha sp., Mesosphaerum pectinatum, $M$. urundeuva, $P$. granata and Rosmarinus officinalis. Of these, only $A$. occidentale and $M$. urundeuva are native to Brazil (Table No. 1). 
Table No. 1

Species cited by Roma informants living in the Agreste (Alves family) and northern Atlantic forest (Dantas family) of Pernambuco. Categories of use: food (f), cosmetic $(\mathrm{Cm})$, condiment $(\mathrm{Cd})$, medicinal (M), ritualistic $(R)$, technological (T), veterinary (V), and others (O). Number of informants who cited the plant (NIC); Number of citations (NC); Number of uses (NU); Use value (UV), International Statistical Classification of Diseases and Related Health Problems (ICD)

\begin{tabular}{|c|c|c|c|c|c|c|c|c|c|}
\hline Families & Species & $\begin{array}{l}\text { Common } \\
\text { name }\end{array}$ & Part used & NIC & NC & NU & $\mathbf{U V}$ & Uses & $\begin{array}{l}\text { ICD (when } \\
\text { medicinal }\end{array}$ \\
\hline $\begin{array}{l}\text { Acanthaceae } \\
\text { Juss. }\end{array}$ & Justicia pectoralis Jacq & Anador & Leaf & 4 & 4 & 3 & 0.13 & M & $\begin{array}{l}\text { R05; R51; } \\
\text { R52.0 }\end{array}$ \\
\hline $\begin{array}{l}\text { Adoxaceae E. } \\
\text { Mey. }\end{array}$ & Sambucus nigra L. & $\begin{array}{l}\text { Sabugueiro } \\
\text { (Elderberry) }\end{array}$ & Flower & 4 & 5 & 3 & 0.13 & M & $\begin{array}{l}\text { I10; J11; } \\
\text { J40; R50.9 }\end{array}$ \\
\hline \multirow{4}{*}{$\begin{array}{l}\text { Amaranthaceae } \\
\text { A. Juss. }\end{array}$} & $\begin{array}{l}\text { Alternanthera dentata } \\
\text { (Moench) Stuchlík ex } \\
\text { R.E.Fr. }\end{array}$ & Dipirona & Leaf & 1 & 1 & 1 & 0.04 & M & I10 \\
\hline & Amaranthus blitum $\mathrm{L}$. & $\begin{array}{l}\text { Bredo-de- } \\
\text { porco }\end{array}$ & Leaf & 1 & 1 & 1 & 0.04 & $\mathrm{Cd}$ & --------------- \\
\hline & $\begin{array}{l}\text { Dysphania } \\
\text { ambrosioides (L.) } \\
\text { Mosyakin \& Clemants }\end{array}$ & Mastruz & Leaf & 6 & 9 & 4 & 0.17 & M & $\begin{array}{l}\text { A37.9; } \\
\text { B82.0; } \\
\text { F50.9; J11; } \\
\text { R05; R52.0; } \\
\text { T14.1 }\end{array}$ \\
\hline & $\begin{array}{l}\text { Gomphrena demissa } \\
\text { Mart }\end{array}$ & Capitãozinho & Root & 1 & 1 & 1 & 0.04 & M & $\mathrm{J} 11$ \\
\hline \multirow[t]{2}{*}{$\begin{array}{l}\text { Amaryllidaceae } \\
\text { J. St.-Hil }\end{array}$} & Allium cepa $\mathrm{L}$. & $\begin{array}{l}\text { Cebola } \\
\text { (Onion) }\end{array}$ & All & 5 & 10 & 4 & 0.17 & $\begin{array}{l}\mathrm{F} / \mathrm{Cd} / \\
\mathrm{M}\end{array}$ & $\begin{array}{l}\text { I74, J40; } \\
\text { N94; R05; } \\
\text { R51 }\end{array}$ \\
\hline & Allium sativum $\mathrm{L}$. & Alho (Garlic) & All & 2 & 3 & 2 & 0.09 & $\mathrm{~F} / \mathrm{M}$ & $\mathrm{I} 74$ \\
\hline \multirow{4}{*}{$\begin{array}{l}\text { Anacardiaceae } \\
\text { R. Br. }\end{array}$} & $\begin{array}{l}\text { Anacardium occidentale } \\
\text { L. }\end{array}$ & $\begin{array}{l}\text { Cajueiro } \\
\text { (Cashew) }\end{array}$ & $\begin{array}{l}\text { Stem /Seed / } \\
\text { Pseudofruit }\end{array}$ & 6 & 17 & 5 & 0.22 & $\mathrm{~F} / \mathrm{M}$ & $\begin{array}{l}\text { I10; L98.9; } \\
\text { N71.9; } \\
\text { R52.0; } \\
\text { T14.1 }\end{array}$ \\
\hline & $\begin{array}{l}\text { Myracrodruon } \\
\text { urundeuva (Engl.) Fr. } \\
\text { Allemão }\end{array}$ & Aroeira & Stem & 10 & 14 & 5 & 0.22 & $\mathrm{M} / \mathrm{T}$ & $\begin{array}{l}\text { K05; L98.9; } \\
\text { N39.0; } \\
\text { N76.0; R10; } \\
\text { T14.1 }\end{array}$ \\
\hline & Spondias mombin L. & Cajarana & Fruit & 2 & 2 & 1 & 0.04 & $\mathrm{~F}$ & ---------------- \\
\hline & $\begin{array}{l}\text { Spondias tuberosa } \\
\text { Arruda }\end{array}$ & $\begin{array}{l}\text { Imbu or } \\
\text { Umbu }\end{array}$ & Root & 2 & 2 & 1 & 0.04 & $\mathrm{~F}$ & --------------- \\
\hline \multirow{3}{*}{$\begin{array}{l}\text { Annonaceae } \\
\text { Juss. }\end{array}$} & \multirow{2}{*}{$\begin{array}{l}\text { Annona coriacea Mart. } \\
\text { Annona muricata } \mathrm{L} .\end{array}$} & \multirow{3}{*}{$\begin{array}{l}\text { Aticum } \\
\text { Graviola } \\
\text { (Soursop) } \\
\text { Pinha } \\
\text { (Custard } \\
\text { apple) }\end{array}$} & Leaf & 2 & 2 & 2 & 0.09 & M & I64; E78.0 \\
\hline & & & Leaf/Fruit & 2 & 4 & 3 & 0.13 & $\mathrm{~F} / \mathrm{M}$ & E78.0; N41 \\
\hline & Annona squamosa $\mathrm{L}$. & & Fruit/Seed & 2 & 2 & 2 & 0.09 & $\mathrm{~F} / \mathrm{M}$ & B85.2 \\
\hline \multirow[t]{2}{*}{ Apiaceae Lindl. } & Coriandrum sativum $\mathrm{L}$. & $\begin{array}{l}\text { Coentro } \\
\text { (Coriander) }\end{array}$ & $\begin{array}{l}\text { Stem/Leaf } \\
\text { /Root/Seed }\end{array}$ & 5 & 11 & 5 & 0.22 & $\begin{array}{l}\mathrm{F} / \mathrm{Cd} / \\
\mathrm{M} / \mathrm{R}\end{array}$ & $\begin{array}{l}\text { E78.0; } \\
\text { H83.0; N41; } \\
\text { R10 }\end{array}$ \\
\hline & $\begin{array}{l}\text { Foeniculum vulgare } \\
\text { Mill. }\end{array}$ & $\begin{array}{l}\text { Erva-doce } \\
\text { (Fennel) }\end{array}$ & Seed & 4 & 6 & 4 & 0.17 & $\begin{array}{l}\mathrm{Cd} / \mathrm{M} / \\
\mathrm{R}\end{array}$ & $\begin{array}{l}\text { J11; R10; } \\
\text { R11 }\end{array}$ \\
\hline $\begin{array}{l}\text { Apocynaceae } \\
\text { Juss. }\end{array}$ & $\begin{array}{l}\text { Aspidosperma } \\
\text { pyrifolium Mart. \& } \\
\text { Zucc. }\end{array}$ & $\begin{array}{l}\text { Pereiro-da- } \\
\text { caatinga }\end{array}$ & Stem & 2 & 2 & 2 & 0.09 & $\mathrm{M} / \mathrm{V}$ & M54.6 \\
\hline \multirow{3}{*}{$\begin{array}{l}\text { Arecaceae } \\
\text { Schultz Sch. }\end{array}$} & \multirow{3}{*}{$\begin{array}{l}\text { Acrocomia aculeata } \\
\text { (Jacq.) Lodd. Ex Mart. } \\
\text { Attalea compta Mart. }\end{array}$} & Macaíba & Fruit & 1 & 1 & 1 & 0.04 & M & $\mathrm{J} 40$ \\
\hline & & \multirow{2}{*}{$\begin{array}{l}\text { Catolé } \\
\text { Coco } \\
\text { (Coconut) }\end{array}$} & Fruit & 1 & 1 & 1 & 0.04 & \multirow{2}{*}{$\begin{array}{l}\mathrm{M} \\
\mathrm{F} / \mathrm{Cm} / \\
\mathrm{M}\end{array}$} & \multirow{2}{*}{$\begin{array}{l}\mathrm{H} 25 \\
\text { A37.9; } \\
\text { K77.0; H10; }\end{array}$} \\
\hline & & & Fruit & 6 & 13 & 5 & 0.22 & & \\
\hline
\end{tabular}




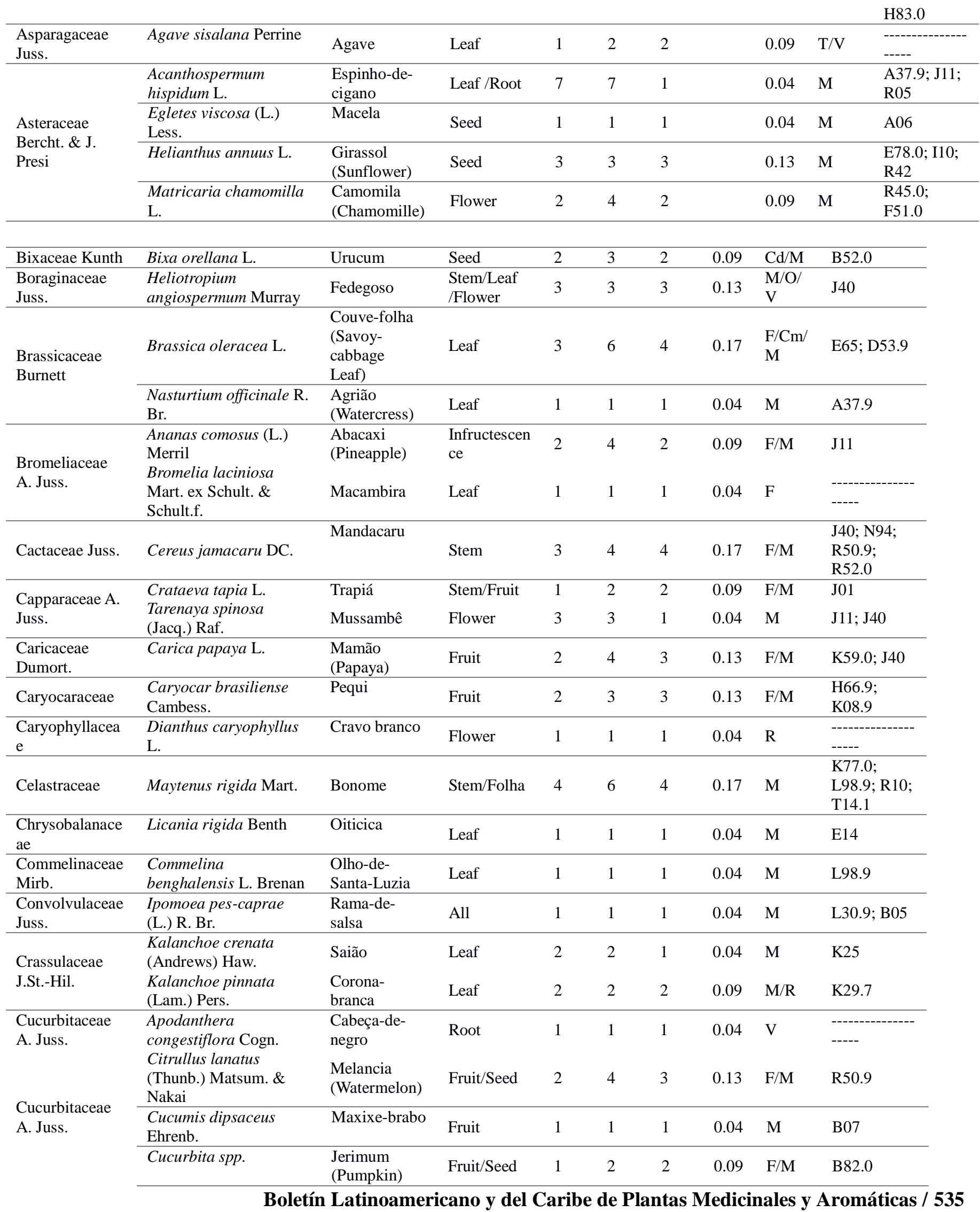




\begin{tabular}{|c|c|c|c|c|c|c|c|c|c|}
\hline & Luffa operculata Cogn. & $\begin{array}{l}\text { Cabacinha or } \\
\text { buchinha }\end{array}$ & Fruit & 3 & 3 & 2 & 0.09 & $\mathrm{O}$ & J01; O06 \\
\hline & $\begin{array}{l}\text { Momordica charantia } \\
\text { L. }\end{array}$ & Melão-brabo & Stem/Leaf & 2 & 2 & 2 & 0.09 & $\mathrm{M} / \mathrm{V}$ & I84 \\
\hline & $\begin{array}{l}\text { Sechium edule (Jacq.) } \\
\text { Swartz }\end{array}$ & $\begin{array}{l}\text { Chuchu } \\
\text { (Chayote) }\end{array}$ & Fruit & 2 & 4 & 2 & 0.09 & $\mathrm{~F} / \mathrm{M}$ & I10 \\
\hline $\begin{array}{l}\text { Dioscoreaceae } \\
\text { R. Br. }\end{array}$ & Dioscorea trifida L. f. & Inhame (Yam) & Stem & 3 & 4 & 2 & 0.09 & $\mathrm{~F} / \mathrm{M}$ & M25.5 \\
\hline \multirow{5}{*}{$\begin{array}{l}\text { Euphorbiaceae } \\
\text { Juss. }\end{array}$} & \multirow{2}{*}{$\begin{array}{l}\text { Cnidoscolus urens (L.) } \\
\text { Croton micans Sw. }\end{array}$} & \multirow{2}{*}{$\begin{array}{l}\text { Urtiga-branca } \\
\text { Velame- } \\
\text { branco }\end{array}$} & Root & 1 & 1 & 1 & 0.04 & M & K29.7 \\
\hline & & & Stem & 1 & 1 & 1 & 0.04 & M & L98.9 \\
\hline & Croton urticifolius Lam. & $\begin{array}{l}\text { Marmeleiro- } \\
\text { branco }\end{array}$ & Leaf & 1 & 1 & 1 & 0.04 & $\mathrm{M}$ & R10 \\
\hline & Euphorbia tirucalli L. & $\begin{array}{l}\text { Avelós or } \\
\text { dedo-do-cão }\end{array}$ & Stem & 3 & 3 & 3 & 0.13 & M & B07; C80 \\
\hline & $\begin{array}{l}\text { Jatropha gossypiifolia } \\
\text { L. }\end{array}$ & Pinhão-roxo & All & 7 & 9 & 3 & 0.13 & $\mathrm{M} / \mathrm{R}$ & R51; T14.1 \\
\hline \multirow{4}{*}{$\begin{array}{l}\text { Euphorbiaceae } \\
\text { Juss. }\end{array}$} & $\begin{array}{l}\text { Jatropha molissima } \\
\text { (Pohl) Baill. }\end{array}$ & Pinhão-brabo & Stem & 1 & 1 & 1 & 0.04 & M & T14.1 \\
\hline & $\begin{array}{l}\text { Manihot esculenta } \\
\text { Crantz }\end{array}$ & $\begin{array}{l}\text { Mandioca } \\
\text { (Cassava) }\end{array}$ & Leaf & 2 & 3 & 2 & 0.09 & $\mathrm{~F} / \mathrm{O}$ & --------------- \\
\hline & Ricinus communis L. & Mamona & Stem/Leaf & 2 & 2 & 2 & 0.09 & M & R51 \\
\hline & $\begin{array}{l}\text { Sapium glandulosum } \\
\text { (L.) Morong }\end{array}$ & Burra-leiteira & Stem & 1 & 1 & 1 & 0.04 & $\mathrm{~T}$ & -------------- \\
\hline \multirow{17}{*}{ Fabaceae Lindl. } & $\begin{array}{l}\text { Amburana cearensis } \\
\text { (Allemão) A. C. Sm. }\end{array}$ & $\begin{array}{l}\text { Imburama-de- } \\
\text { cheiro }\end{array}$ & Stem & 3 & 3 & 3 & 0.13 & $\mathrm{M} / \mathrm{T}$ & L98.9; R05 \\
\hline & $\begin{array}{l}\text { Anadenanthera } \\
\text { colubrina (Vell.) } \\
\text { Benan }\end{array}$ & Angico & Stem & 4 & 5 & 4 & 0.17 & $\mathrm{M} / \mathrm{T}$ & $\begin{array}{l}\text { J40; R53; } \\
\text { T14.1 }\end{array}$ \\
\hline & $\begin{array}{l}\text { Bauhinia cheilantha } \\
\text { (Bong.) Steud. }\end{array}$ & Mororó & Stem & 2 & 3 & 3 & 0.13 & $\mathrm{M} / \mathrm{T}$ & $\begin{array}{l}\text { L98.9; } \\
\text { M54.6 }\end{array}$ \\
\hline & Bauhinia sp. & \multirow{2}{*}{$\begin{array}{l}\text { Pata-de-vaca } \\
\text { Feijão-guandu }\end{array}$} & Leaf & 2 & 2 & 2 & 0.09 & $\mathrm{Cm} / \mathrm{M}$ & E14 \\
\hline & $\begin{array}{l}\text { Cajanus cajan (L.) } \\
\text { Huth }\end{array}$ & & Seed & 2 & 2 & 1 & 0.04 & M & I10 \\
\hline & $\begin{array}{l}\text { Desmanthus virgatus } \\
\text { (L.) Willd }\end{array}$ & Jureminha & Leaf & 1 & 1 & 1 & 0.04 & $\mathrm{R}$ & ------ \\
\hline & $\begin{array}{l}\text { Erythrina velutina } \\
\text { Willd }\end{array}$ & Mulungu & Stem & 2 & 3 & 2 & 0.09 & $\mathrm{M} / \mathrm{T}$ & R60 \\
\hline & $\begin{array}{l}\text { Hymenaea courbaril } \\
\text { L. }\end{array}$ & Jatobá & Fruit & 1 & 1 & 1 & 0.04 & M & $\mathrm{J} 40$ \\
\hline & $\begin{array}{l}\text { Libidibia ferrea } \\
\text { (Mart. ex Tul.) } \\
\text { L.P.Queiroz }\end{array}$ & Jucá & Fruit & 2 & 2 & 1 & 0.04 & M & $\begin{array}{l}\text { L98.9; } \\
\text { R52.0 }\end{array}$ \\
\hline & $\begin{array}{l}\text { Melanoxylon brauna } \\
\text { Schoot }\end{array}$ & Braúna & Stem & 2 & 2 & 2 & 0.09 & $\mathrm{~T}$ & ---------------- \\
\hline & $\begin{array}{l}\text { Mimosa } \\
\text { caesalpiniifolia } \\
\text { Benth. }\end{array}$ & Sabiá & Stem/Leaf & 2 & 3 & 2 & 0.09 & $\mathrm{M} / \mathrm{T}$ & E78.0 \\
\hline & Mimosa pudica L. & Malícia & Root & 3 & 3 & 2 & 0.09 & M & $\begin{array}{l}\text { D53.9; } \\
\text { K77.0 }\end{array}$ \\
\hline & $\begin{array}{l}\text { Mimosa tenuiflora } \\
\text { (Willd.) Poir. }\end{array}$ & Jurema-preta & Stem/Root & 4 & 6 & 4 & 0.17 & $\mathrm{M} ; \mathrm{R}$ & $\begin{array}{l}\text { K08.9; K30; } \\
\text { L98.9; } \\
\text { N39.0 }\end{array}$ \\
\hline & Phaseolus lunatus L. & Fava & Stem/Leaf & 2 & 4 & 3 & 0.13 & $\mathrm{~F} / \mathrm{M} / \mathrm{V}$ & B36.0 \\
\hline & $\begin{array}{l}\text { Poincianella } \\
\text { microphylla (Mart. ex } \\
\text { G.Don) L.P.Queiroz } \\
\text { Poincianella }\end{array}$ & $\begin{array}{l}\text { Catingueira- } \\
\text { rasteira }\end{array}$ & $\begin{array}{l}\text { Stem/Flowe } \\
\mathrm{r}\end{array}$ & 3 & 3 & 2 & 0.09 & M & $\begin{array}{l}\text { L98.9; } \\
\text { R52.0; R60 }\end{array}$ \\
\hline & $\begin{array}{l}\text { pyramidalis (Tul.) } \\
\text { L.P.Queiroz }\end{array}$ & Catingueira & Flower & 3 & 3 & 2 & 0.09 & M & $\mathrm{J} 11$ \\
\hline & Prosopis juliflora DC. & Algaroba & Seed & 2 & 2 & 1 & 0.04 & $\mathrm{~F}$ & --------------- \\
\hline
\end{tabular}




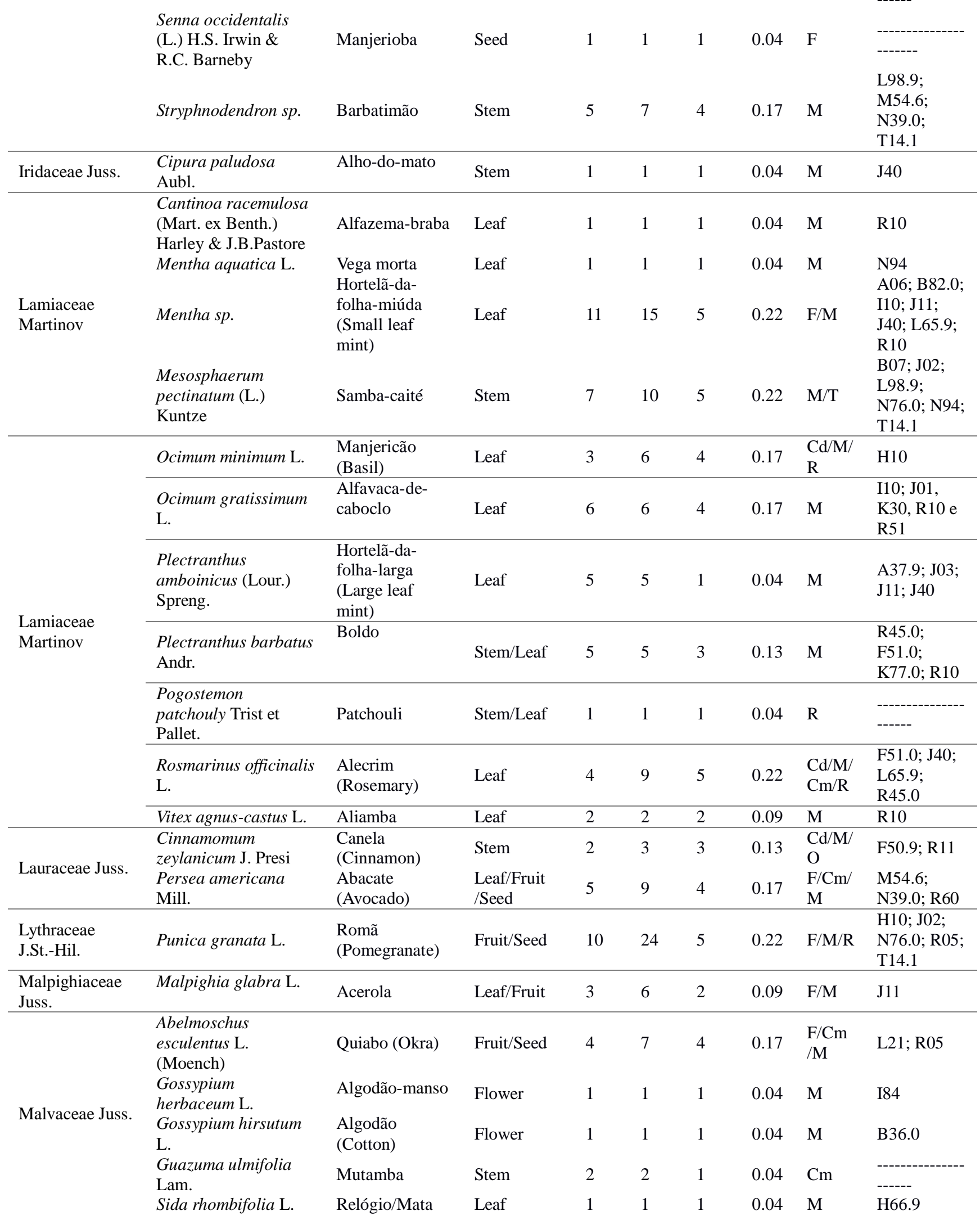




\begin{tabular}{|c|c|c|c|c|c|c|c|c|c|}
\hline Malvaceae Juss. & Urena lobata $\mathrm{L}$. & $\begin{array}{l}\text { pasto } \\
\text { Malva-rosa }\end{array}$ & Leaf & 1 & 1 & 1 & 0.04 & M & J11 \\
\hline $\begin{array}{l}\text { Meliaceae A. } \\
\text { Juss. }\end{array}$ & $\begin{array}{l}\text { Azadirachta indica A. } \\
\text { Juss. }\end{array}$ & Nim & All & 2 & 2 & 1 & 0.04 & $\mathrm{O}$ & $\begin{array}{l}-------------- \\
------\end{array}$ \\
\hline $\begin{array}{l}\text { Moraceae } \\
\text { Gaudich }\end{array}$ & $\begin{array}{l}\text { Artocarpus altilis } \\
\text { (Parkinson) Fosberg }\end{array}$ & $\begin{array}{l}\text { Fruta-pão } \\
\text { (Breadfruit) }\end{array}$ & Leaf/Fruit & 2 & 3 & 2 & 0.09 & M & R60 \\
\hline Musaceae Juss. & Musa spp. & Banana & Flower/Fruit & 6 & 13 & 4 & 0.17 & $\mathrm{~F} / \mathrm{M}$ & $\begin{array}{l}\text { A37.9; L21; } \\
\text { R05; R60; } \\
\text { T14.1 }\end{array}$ \\
\hline \multirow{6}{*}{ Myrtaceae Juss. } & Eucalyptus sp. & $\begin{array}{l}\text { Eucalipto-da } \\
\text { folha-fina } \\
\text { (Narrow leaf } \\
\text { eucalyptus) }\end{array}$ & Leaf & 7 & 7 & 3 & 0.13 & M & $\begin{array}{l}\text { J01; R50.9; } \\
\text { R51 }\end{array}$ \\
\hline & Eugenia uniflora L. & $\begin{array}{l}\text { Pitangueira } \\
\text { (Brazilian } \\
\text { cherry tree) }\end{array}$ & Leaf/Fruit & 3 & 6 & 2 & 0.09 & $\mathrm{~F} / \mathrm{M}$ & $\mathrm{R} 10$ \\
\hline & Psidium guajava L. & $\begin{array}{l}\text { Goiabeira } \\
\text { (Guava) }\end{array}$ & Leaf & 4 & 7 & 2 & 0.09 & M & $\mathrm{R} 10$ \\
\hline & $\begin{array}{l}\text { Syzygium aqueum } \\
\text { (Burm.f.) Alston }\end{array}$ & $\begin{array}{l}\text { Jambo-branco } \\
\text { (Jambu air) }\end{array}$ & Leaf & 1 & 1 & 1 & 0.04 & M & E14 \\
\hline & $\begin{array}{l}\text { Syzygium aromaticum } \\
\text { L. }\end{array}$ & $\begin{array}{l}\text { Cravo-da-índia } \\
\text { (Clove) }\end{array}$ & Flower & 1 & 2 & 2 & 0.09 & $\mathrm{Cd} / \mathrm{M}$ & $\mathrm{R} 10$ \\
\hline & $\begin{array}{l}\text { Syzygium cumini (L.) } \\
\text { Skeels }\end{array}$ & $\begin{array}{l}\text { Azeitona-preta } \\
\text { (Black olive) / } \\
\text { Jamelão } \\
\text { (Jambolan) }\end{array}$ & Leaf & 2 & 2 & 1 & 0.04 & M & E78.0 \\
\hline $\begin{array}{l}\text { Nyctaginaceae } \\
\text { Juss. }\end{array}$ & Boerhavia diffusa $\mathrm{L}$. & Pega-pinto & Root & 1 & 1 & 1 & 0.04 & M & R33 \\
\hline $\begin{array}{l}\text { Olacaceae R. } \\
\text { Br. }\end{array}$ & $\begin{array}{l}\text { Ximenia americana } \\
\text { L. }\end{array}$ & $\begin{array}{l}\text { Ameixa-do- } \\
\text { mato (Wild } \\
\text { plum) }\end{array}$ & Stem & 2 & 2 & 2 & 0.09 & M & L98.9 \\
\hline $\begin{array}{l}\text { Oxalidaceae R. } \\
\text { Br. }\end{array}$ & $\begin{array}{l}\text { Averrhoa } \\
\text { carambola } \mathrm{L} .\end{array}$ & $\begin{array}{l}\text { Carambola } \\
\text { (Star fruit) }\end{array}$ & Leaf & 2 & 2 & 2 & 0.09 & $\mathrm{~F} / \mathrm{M}$ & N39.0 \\
\hline $\begin{array}{l}\text { Oxalidaceae R. } \\
\text { Br. }\end{array}$ & Oxalis sp. & Azedinha & Fruit & 2 & 2 & 1 & 0.04 & $\mathrm{~F}$ & --------------- \\
\hline \multirow[t]{2}{*}{$\begin{array}{l}\text { Passifloraceae } \\
\text { Juss. ex Roussel }\end{array}$} & $\begin{array}{l}\text { Passiflora } \\
\text { cincinnati Mast. }\end{array}$ & $\begin{array}{l}\text { Maracujá-da- } \\
\text { Caatinga } \\
\text { (Passion fruit } \\
\text { from } \\
\text { Caatinga) }\end{array}$ & Fruit & 1 & 1 & 1 & 0.04 & V & --------------- \\
\hline & $\begin{array}{l}\text { Passiflora edulis } \\
\text { Sims }\end{array}$ & $\begin{array}{l}\text { Maracujá } \\
\text { (Passion fruit) }\end{array}$ & Leaf/Fruit & 2 & 4 & 3 & 0.13 & $\mathrm{~F} / \mathrm{M}$ & $\begin{array}{l}\text { R45.0; } \\
\text { F51.0 }\end{array}$ \\
\hline $\begin{array}{l}\text { Pedaliaceae R. } \\
\text { Br. }\end{array}$ & $\begin{array}{l}\text { Sesamum indicum } \\
\text { L. }\end{array}$ & $\begin{array}{l}\text { Gergelim- } \\
\text { preto (Black } \\
\text { sesame) }\end{array}$ & Seed & 1 & 1 & 1 & 0.04 & M & E78.0; I10 \\
\hline $\begin{array}{l}\text { Phytolaccaceae } \\
\text { R. Br. }\end{array}$ & Petiveria alliacea $\mathrm{L}$. & Atipim & Leaf & 1 & 2 & 2 & 0.09 & $\mathrm{M} / \mathrm{R}$ & R52.0 \\
\hline $\begin{array}{l}\text { Phyllanthaceae } \\
\text { Martinov }\end{array}$ & $\begin{array}{l}\text { Phyllanthus amarus } \\
\text { Schumach }\end{array}$ & $\begin{array}{l}\text { Quebra-pedra } \\
\text { (Stone } \\
\text { breaker) }\end{array}$ & Root & 5 & 5 & 1 & 0.04 & M & $\mathrm{N} 20.9$ \\
\hline $\begin{array}{l}\text { Piperaceae } \\
\text { Giseke }\end{array}$ & Piper nigrum L. & $\begin{array}{l}\text { Pimenta-negra } \\
\text { (Black } \\
\text { pepper) }\end{array}$ & Fruit & 2 & 3 & 2 & 0.09 & $\mathrm{Cd} / \mathrm{M}$ & R05 \\
\hline \multirow[t]{2}{*}{$\begin{array}{l}\text { Poaceae } \\
\text { Barnhart }\end{array}$} & $\begin{array}{l}\text { Cymbopogon } \\
\text { citratus D.C Stapf }\end{array}$ & $\begin{array}{l}\text { Capim-santo } \\
\text { (Lemon grass) }\end{array}$ & Leaf & 15 & 21 & 6 & 0.26 & $\mathrm{Cd} / \mathrm{M} / \mathrm{R}$ & $\begin{array}{l}\text { F51.0; I10; } \\
\text { K30; K45; } \\
\text { L65.9; R10; } \\
\text { R45.0 }\end{array}$ \\
\hline & $\begin{array}{l}\text { Echinochlola } \\
\text { crusgalli L. }\end{array}$ & Senha & Seed & 1 & 2 & 2 & 0.09 & $\mathrm{M} / \mathrm{V}$ & K77.0 \\
\hline
\end{tabular}




\begin{tabular}{|c|c|c|c|c|c|c|c|c|c|}
\hline & $\begin{array}{l}\text { Phalaris } \\
\text { canariensis L. }\end{array}$ & $\begin{array}{l}\text { Alpiste } \\
\text { (Canary grass) }\end{array}$ & $\begin{array}{l}\text { Seed } \\
\text { Infructescence }\end{array}$ & 2 & 2 & 2 & $\begin{array}{l}0.09 \\
0.04\end{array}$ & $M$ & $\begin{array}{l}\text { E14; K77.0 } \\
\text { K77 }\end{array}$ \\
\hline & & & Infructescence & 2 & 3 & 2 & 0.04 & $\mathrm{M}$ & K77.0 \\
\hline Portulaceae Juss & $\begin{array}{l}\text { Talinum racemosum } \\
\text { (Jacq.) Willd. }\end{array}$ & $\begin{array}{l}\text { Bredo-major- } \\
\text { gomes }\end{array}$ & Leaf & 1 & 1 & 1 & 0.04 & $\mathrm{~F}$ & --------------- \\
\hline $\begin{array}{l}\text { Rhamnaceae } \\
\text { Juss. }\end{array}$ & $\begin{array}{l}\text { Ziziphus joazeiro } \\
\text { Mart. }\end{array}$ & Juá & Stem/Fruit & 2 & 4 & 3 & 0.13 & $\mathrm{~F} / \mathrm{Cm}$ & --------------- \\
\hline \multirow{2}{*}{ Rosaceae Juss. } & Malus pumila Mill. & Maçã (Apple) & Pseudofruit & 2 & 4 & 3 & 0.13 & $\begin{array}{l}\mathrm{F} / \mathrm{M} \\
/ \mathrm{Cm}\end{array}$ & $\mathrm{J} 02$ \\
\hline & Rosa spp. & Rosa (Rose) & Flower & 5 & 5 & 1 & 0.04 & $\mathrm{R}$ & --------------- \\
\hline \multirow{5}{*}{ Rubiaceae Juss. } & $\begin{array}{l}\text { Borreria verticillata } \\
\text { (L.) G. Mev. }\end{array}$ & $\begin{array}{l}\text { Vassourinha- } \\
\text { de-botão }\end{array}$ & Root & 5 & 5 & 4 & 0.17 & M & $\begin{array}{l}\text { I84; N39.0; } \\
\text { N41; N76.0 }\end{array}$ \\
\hline & $\begin{array}{l}\text { Coutarea hexandra } \\
\text { (Jacq.) K.Schum. }\end{array}$ & Quina-quina & Stem & 1 & 1 & 1 & 0.04 & M & L98.9 \\
\hline & $\begin{array}{l}\text { Genipa americana } \\
\text { L. }\end{array}$ & Jenipapo & Fruit & 1 & 1 & 1 & 0.04 & M & R05 \\
\hline & $\begin{array}{l}\text { Morinda citrifolia } \\
\text { L. }\end{array}$ & Noni & Fruit & 1 & 1 & 1 & 0.04 & M & $\begin{array}{l}\text { E14; N41; } \\
\text { N71.9 }\end{array}$ \\
\hline & $\begin{array}{l}\text { Uncaria tomentosa } \\
\text { (Willd. Ex Roem. \& } \\
\text { Schult) DC. }\end{array}$ & Unha-de-gato & Stem & 1 & 1 & 1 & 0.04 & M & M54.6 \\
\hline \multirow{3}{*}{$\begin{array}{l}\text { Rutaceae A. } \\
\text { Juss. }\end{array}$} & $\begin{array}{l}\text { Citrus limonum (L.) } \\
\text { Osbeck }\end{array}$ & $\begin{array}{l}\text { Limão } \\
\text { (Lemon) }\end{array}$ & Fruit & 3 & 6 & 4 & 0.17 & $\begin{array}{l}\mathrm{F} / \mathrm{Cm} \\
/ \mathrm{M}\end{array}$ & J01; R52.0 \\
\hline & Citrus spp. & $\begin{array}{l}\text { Laranja } \\
\text { (Orange) }\end{array}$ & Leaf/Fruit & 6 & 12 & 3 & 0.13 & $\mathrm{~F} / \mathrm{M}$ & $\begin{array}{l}\text { F51.0; J40; } \\
\text { R45.0 }\end{array}$ \\
\hline & Ruta graveolens L. & Arruda (Rue) & Leaf & 6 & 6 & 2 & 0.09 & $\mathrm{M} / \mathrm{R}$ & H66.9; R42 \\
\hline $\begin{array}{l}\text { Sapindaceae } \\
\text { Juss. }\end{array}$ & $\begin{array}{l}\text { Sapindus saponaria } \\
\text { L. }\end{array}$ & Saboneteira & Fruit & 1 & 1 & 1 & 0.04 & $\mathrm{Cm}$ & \\
\hline $\begin{array}{l}\text { Sapotaceae } \\
\text { Juss. }\end{array}$ & $\begin{array}{l}\text { Chrysophyllum } \\
\text { imperiale (Linden } \\
\text { ex K.Koch \& } \\
\text { Fintelm.) }\end{array}$ & $\begin{array}{l}\text { Marmeleiro- } \\
\text { do-mato (Wild } \\
\text { quince) }\end{array}$ & Stem & 1 & 2 & 2 & 0.09 & $\mathrm{R}$ & -------------- \\
\hline Sapotaceae Juss & $\begin{array}{l}\text { Sideroxylum } \\
\text { obtusifolium } \\
\text { (Roem. E Schult.) } \\
\text { T. D. Penn. }\end{array}$ & Quixaba & Stem & 4 & 4 & 2 & 0.09 & M & $\mathrm{R} 52.0$ \\
\hline $\begin{array}{l}\text { Schisandraceae } \\
\text { Bl. }\end{array}$ & $\begin{array}{l}\text { Illicium verum } \\
\text { Hook } \mathrm{f} .\end{array}$ & $\begin{array}{l}\text { Anis-estrelado } \\
\text { (Star anise) }\end{array}$ & Flower/Seed & 2 & 2 & 1 & 0.04 & M & $\mathrm{R} 10$ \\
\hline \multirow{7}{*}{$\begin{array}{l}\text { Solanaceae A. } \\
\text { Juss. }\end{array}$} & $\begin{array}{l}\text { Brugmansia } \\
\text { suaveolens } \\
\text { (Willd.) Sweet }\end{array}$ & Zabumba & Leaf & 1 & 1 & 1 & 0.04 & M & $\mathrm{J} 45$ \\
\hline & $\begin{array}{l}\text { Capsicum } \\
\text { frutescens } \mathrm{L} .\end{array}$ & $\begin{array}{l}\text { Pimenta- } \\
\text { malagueta } \\
\text { (Chilli } \\
\text { pepper) }\end{array}$ & Leaf & 2 & 3 & 2 & 0.09 & M & R51 \\
\hline & $\begin{array}{l}\text { Nicotiana } \\
\text { tabacum } \mathrm{L} .\end{array}$ & $\begin{array}{l}\text { Fumo } \\
\text { (Tobacco) }\end{array}$ & Flower/Leaf & 2 & 2 & 2 & 0.09 & $\mathrm{~V}$ & $----\cdot$ \\
\hline & Solanum sp. & Gogoia & Fruit/Root & 2 & 2 & 2 & 0.09 & $\mathrm{~F} / \mathrm{M}$ & N39.0 \\
\hline & $\begin{array}{l}\text { Solanum nigrum } \\
\text { L. }\end{array}$ & Erva moura & Leaf/Root & 4 & 4 & 2 & 0.09 & M & $\begin{array}{l}\text { K08.9; N39.0; } \\
\text { N94 }\end{array}$ \\
\hline & $\begin{array}{l}\text { Solanum } \\
\text { paniculatum L. }\end{array}$ & Jurubeba & $\begin{array}{l}\text { Stem/Fruit } \\
\text { /Root }\end{array}$ & 4 & 7 & 4 & 0.17 & $\begin{array}{l}\mathrm{Cm} / \mathrm{M} \\
/ \mathrm{V}\end{array}$ & D53.9; J11; J40 \\
\hline & $\begin{array}{l}\text { Solanum } \\
\text { tuberosum L. }\end{array}$ & $\begin{array}{l}\text { Batata-inglesa } \\
\text { (Potato) }\end{array}$ & Stem/Leaf & 3 & 5 & 3 & 0.13 & $\mathrm{Cm} / \mathrm{M}$ & $\mathrm{K} 29.7$ \\
\hline $\begin{array}{l}\text { Turneraceae } \\
\text { Kunth ex DC. }\end{array}$ & $\begin{array}{l}\text { Turnera subulata } \\
\text { Sm. }\end{array}$ & Chanana & Flower & 2 & 2 & 1 & 0.04 & M & A37.9 \\
\hline Urticaceae Juss. & $\begin{array}{l}\text { Cecropia } \\
\text { pachysrachya } \\
\text { Trécul. }\end{array}$ & Imbaúba & Stem & 2 & 2 & 2 & 0.09 & M & L80; M54.6 \\
\hline
\end{tabular}




\begin{tabular}{|c|c|c|c|c|c|c|c|c|c|}
\hline \multirow[b]{2}{*}{$\begin{array}{l}\text { Verbenaceae } \\
\text { J.St.-Hil. }\end{array}$} & $\begin{array}{l}\text { Lantana camara } \\
\text { L. }\end{array}$ & $\begin{array}{l}\text { Erva- } \\
\text { chumbinho }\end{array}$ & Flower & 1 & 1 & 1 & 0.04 & M & L37.9 \\
\hline & $\begin{array}{l}\text { Lippia alba (Mill.) } \\
\text { N.E.Br. ex P. } \\
\text { Wilson }\end{array}$ & Erva-cidreira & $\begin{array}{l}\text { Leaf/ } \\
\text { Seed }\end{array}$ & 10 & 14 & 5 & 0.22 & $\mathrm{M} / \mathrm{V}$ & $\begin{array}{l}\text { D53.9; F51.0; } \\
\text { K30; K59; R10; } \\
\text { R11; R45.0; R51 }\end{array}$ \\
\hline Violaceae Batsch & $\begin{array}{l}\text { Hybanthus cf. } \\
\text { ipepacuanha (L.) } \\
\text { Baill }\end{array}$ & Pepaconha & Root & 2 & 2 & 2 & 0.09 & M & K08; J11 \\
\hline Vitaceae Juss. & $\begin{array}{l}\text { Cissus verticillata } \\
\text { (L.) Nicolson \& } \\
\text { C.E. Jarvis }\end{array}$ & $\begin{array}{l}\text { Insulina } \\
\text { (Insulin) }\end{array}$ & Leaf/Fruit & 2 & 3 & 1 & 0.04 & M & E14 \\
\hline $\begin{array}{l}\text { Xanthorrhoeaceae } \\
\text { Dumort }\end{array}$ & $\begin{array}{l}\text { Aloe vera }(L .) \\
\text { Burm. f. }\end{array}$ & Babosa & Leaf & 9 & 14 & 4 & 0.17 & $\mathrm{Cm} / \mathrm{M}$ & $\begin{array}{l}\text { B65.1; B82.0; } \\
\text { I84; T14.1 }\end{array}$ \\
\hline \multirow{2}{*}{$\begin{array}{l}\text { Zingiberaceae } \\
\text { Martinov }\end{array}$} & $\begin{array}{l}\text { Alpinia zerumbet } \\
\text { (Pers.) Burtt \& R. } \\
\text { M. Smith }\end{array}$ & $\begin{array}{l}\text { Colônia } \\
\text { (Colony) }\end{array}$ & Flower/Leaf & 5 & 9 & 4 & 0.17 & $\mathrm{M} / \mathrm{R}$ & $\begin{array}{l}\text { F51.0; J01; J02; } \\
\text { J40; R45.0; } \\
\text { R50.9; R51 }\end{array}$ \\
\hline & $\begin{array}{l}\text { Zingiber officinale } \\
\text { Roscoe }\end{array}$ & $\begin{array}{l}\text { Gengibre } \\
\text { (Ginger) }\end{array}$ & Root & 4 & 7 & 4 & 0.17 & $\mathrm{Cd} / \mathrm{C} / \mathrm{M}$ & E65/N94 \\
\hline
\end{tabular}

The respondents made 620 citations of uses of the 157 plant species listed in Table No. 1. Members of the Dantas family (Figure No. 2) contributed with 202 citations (97 species, 54 families) and those of the Alves family (Figure No. 3) with 418 citations (139 species, 58 families). Eightyone species were cited by both Roma families, leading to a similarity index of 0.68 in their useful flora. The similarity rises to 0.80 when the 10 most cited plants are compared, both in terms of species and indications of use. Most of the informants had a good knowledge of the use of xacas (herbs in chib), but three chiefs, two from the Dantas family and one from the Alves family, stood out. They were called "pajés" (shaman) by the other Roma people due to their extensive knowledge about medicinal species.

A comparison between the set of plants used by the Dantas family and the lists of useful species found in ethnobotanical surveys of non-Roma communities living in the northern Atlantic forest of Pernambuco (Rodrigues \& Andrade, 2014; Lima, 2015) showed 76 shared species, which corresponds to a similarity index of 0.60 . In turn, among the set of species used by the Dantas family, 89 were present in the lists of useful species provided by surveys of the ethnobotanical knowledge of non-Roma communities living in the Agreste of Pernambuco (Albuquerque et al., 2008; Carvalho et al., 2013), corresponding to a similarity index of 0.68 .

Figure No. 2

Interviewees of the Dantas Roma family in a farm close to their homes in Itambé, northern Atlantic forest of Pernambuco

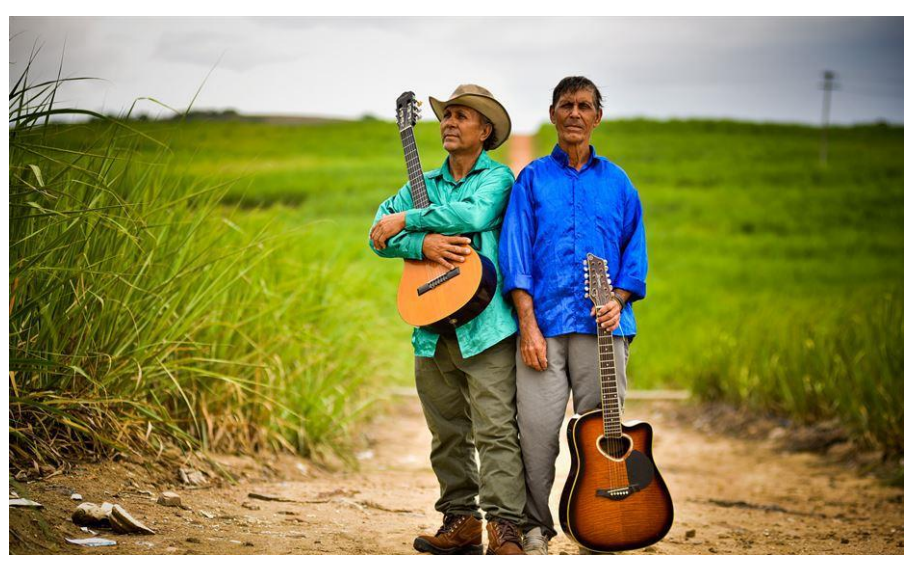

Boletín Latinoamericano y del Caribe de Plantas Medicinales y Aromáticas / 540 
Figure No. 3

Ricardo Lobo accompanied by Enildo Soares (Roma president of ACIPE) and two informants of the Alves family next to one of the houses where they live in the municipality of Altinho, Agreste of Pernambuco

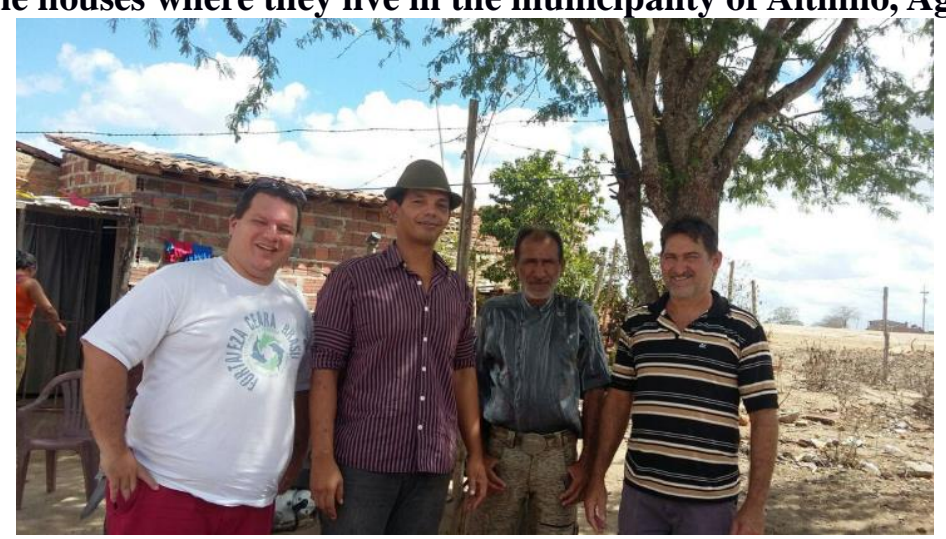

Figure No. 4

Interview with a 102-year-old informant, Alves 6, at her home in Altinho, Agreste of Pernambuco.

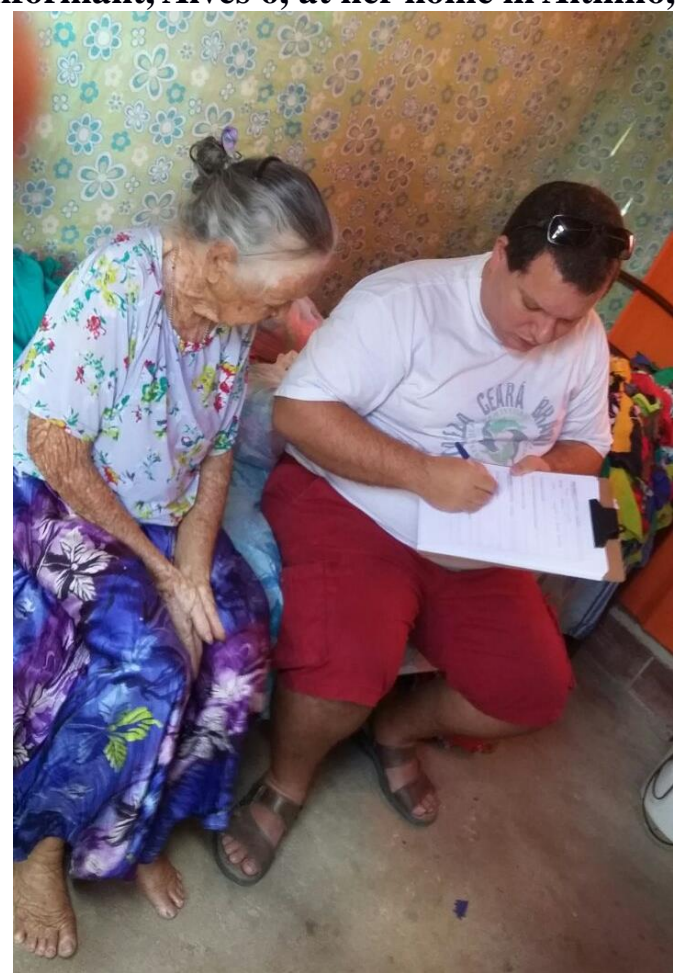

Adults aged 40 to 50 years made 191 citations of use for 78 plant species (native or exotic) belonging to 39 families. Those of the next age group (51 to 60 years) made 259 citations for 121 species belonging to 53 families. Adults over 60 years old made 170 citations for 70 species belonging to 42 families. Twenty-eight species were cited by the three age groups, while 13 species were cited exclusively by informants over 60 (Figure No. 4), but which are commonly use in other non-Roma communities, such as macaíba (Acrocomia aculeata) and juá (Ziziphus joazeiro), native to northeastern Brazil.

As for the sex of the interviewees, Roma men made 356 citations (57\%) of use for 131 species

Boletín Latinoamericano y del Caribe de Plantas Medicinales y Aromáticas / 541 
belonging to 60 families, while Roma women made 264 citations (43\%) of use for 93 species belonging to 37 families (Table No. 2). In the group of men, a greater concentration of knowledge was observed in the age group between 51 and 60 years. Two of the three main informants responsible for the knowledge about medicinal plants were of this age group. In the group of women, there was a more balanced distribution of ethnobotanical knowledge among the age groups.

Table No. 2

Ethnobotanical knowledge of Roma people living in the Agreste (Alves family) and northern Atlantic forest (Dantas family) of Pernambuco, distributed by age group and sex

\begin{tabular}{lccccc}
\hline Age group & $\begin{array}{c}\text { Informants } \\
(\mathbf{n} \mathbf{0})\end{array}$ & $\begin{array}{c}\text { Use citations } \\
(\mathbf{n} \mathbf{0})\end{array}$ & $\begin{array}{c}\text { Families } \\
(\mathbf{n} \mathbf{0})\end{array}$ & $\begin{array}{c}\text { Species } \\
(\mathbf{n} \mathbf{0})\end{array}$ & $\begin{array}{c}\text { Native } \\
(\%)\end{array}$ \\
\hline $40-50$ & \multicolumn{7}{c}{ Men } & & & \\
\hline $51-60$ & 5 & 100 & 30 & 52 & 50 \\
\hline$>60$ & 3 & 180 & 53 & 102 & 58 \\
\hline & 3 & 76 & 28 & 37 & 41 \\
\hline $40-50$ & 5 & Women & & 46 & 35 \\
\hline $51-60$ & 3 & 91 & 27 & 46 & 49 \\
\hline$>60$ & 4 & 79 & 28 & 47 & \\
\hline
\end{tabular}

According to the ICF, the plants that stood out for their medicinal applications were the fruit tree $P$. granata (pomegranate), used to treat infections of the eyes and of the female genital tract (H10; N76.0), heal wounds (T14.1), and treat problems of the respiratory system (J02, R05), and A. hispidum ("espinho-de-cigano"), a ruderal herb, whose importance for treating diseases of the respiratory system (A37.9; J11; R05) was mentioned by all participants (Table No. 1).

Regarding the number of citations, the use value and the level of reliability, $P$. granata, a species of Asian origin with very old reports of use in European countries, stood out as the most important for Roma families, with a reliability index of $100 \%$ (Table No. 3). Cymbopogon citratus (lemon grass), also of Asian origin, the second most cited species and indicated for the treatment of disorders of the nervous (F51; R45), circulatory (I10) and respiratory (K30) systems, general pain (R10), and hair loss (L65.9), had a relatively low reliability index (60\%) despite having reached the highest use value $(0.26)$ (Tables No. 1 and No. 3). 
Table No. 3

Reliability index calculated for the 10 species of plants that were cited by more than $30 \%$ of the Roma informants living in the Agreste (Alves family) and northern Atlantic forest (Dantas family) of Pernambuco

\begin{tabular}{|c|c|c|c|c|}
\hline Specie & Common name & $\begin{array}{l}\text { Number of } \\
\text { informants }\end{array}$ & $\begin{array}{l}\text { Uses } \\
\text { (when medicinal - CIF) }\end{array}$ & $\begin{array}{l}\text { Reliability } \\
\text { index }(\%)\end{array}$ \\
\hline $\begin{array}{l}\text { Cymbopogon citratus } \\
\text { D.C Stapf }\end{array}$ & $\begin{array}{l}\text { Capim santo } \\
\text { (Lemon grass) }\end{array}$ & 15 & $\begin{array}{l}\text { Digestive system (9); Nervous system (5); } \\
\text { Cardiovascular system (3); Disinfectant (2); } \\
\text { Cosmetic (2) }\end{array}$ & 60 \\
\hline Mentha sp. & $\begin{array}{l}\text { Hortelã da folha } \\
\text { miúda (Small leaf } \\
\text { mint) }\end{array}$ & 11 & $\begin{array}{l}\text { Digestive system (7); Respiratory system (4); } \\
\text { Cosmetic (2); Cardiovascular system (1); Food (1) }\end{array}$ & 64 \\
\hline Punica granata L. & $\begin{array}{l}\text { Romã } \\
\text { (Pomegranate) }\end{array}$ & 10 & $\begin{array}{l}\text { Respiratory system (10); Food (10); Sensory } \\
\text { system and pain (1); Genital system (1); Skin (1); } \\
\text { Ritualistic (1) }\end{array}$ & 100 \\
\hline $\begin{array}{l}\text { Lippia alba (Mill) } \\
\text { Brow }\end{array}$ & Erva cidreira & 10 & $\begin{array}{l}\text { Digestive system (8); Nervous system (2); } \\
\text { Hematological and immune system (2); Sensory } \\
\text { system and pain (1); Veterinarian (1) }\end{array}$ & 80 \\
\hline $\begin{array}{l}\text { Myracrodruon } \\
\text { urundeuva (Engl.) Fr. } \\
\text { Allemão }\end{array}$ & Aroeira & 10 & $\begin{array}{l}\text { Digestive system (5); Skin (4); Cardiovascular } \\
\text { system (3); Genital system (1); Technological (1) }\end{array}$ & 50 \\
\hline Aloe vera (L.) Burm. f. & Babosa & 9 & $\begin{array}{l}\text { Cosmetic (6); Cardiovascular system (4); Digestive } \\
\text { system (3); Skin(1) }\end{array}$ & 67 \\
\hline Eucalyptus sp. & $\begin{array}{l}\text { Eucalipto da folha } \\
\text { fina (Narrow leaf } \\
\text { eucalyptus) }\end{array}$ & 7 & $\begin{array}{l}\text { Metabolic and endocrine system (5); Respiratory } \\
\text { system (2); Sensory system and pain (2) }\end{array}$ & 71 \\
\hline $\begin{array}{l}\text { Jatropha gossypiifolia } \\
\text { L. }\end{array}$ & Pinhão roxo & 7 & $\begin{array}{l}\text { Ritualistic (6); Skin (2); Sensory system and pain } \\
\text { (1) }\end{array}$ & 86 \\
\hline $\begin{array}{l}\text { Mesosphaerum } \\
\text { pectinatum (L.) Kuntze }\end{array}$ & Samba caité & 7 & $\begin{array}{l}\text { Skin (4); Digestive system (2); Genital system (2); } \\
\text { Respiratory system (1); Technological (1) }\end{array}$ & 75 \\
\hline $\begin{array}{l}\text { Acanthospermum } \\
\text { hispidum D.C }\end{array}$ & $\begin{array}{l}\text { Espinho-de- } \\
\text { cigano }\end{array}$ & 7 & Respiratory system (7) & 100 \\
\hline
\end{tabular}

In the analysis of the relative importance of the 10 species that were mentioned by more than $30 \%$ of the informants (Table No. 4), it was clear that L. alba was the species with the highest index. This finding corroborates with the other three previously mentioned, because this species also reached a high use value (0.22), good reliability $(80 \%)$, and was the third most cited by the informants.

Table No. 4

Relative importance calculated for the 10 medicinal plant species that were mentioned by more than $30 \%$ of the Roma informants living in the Agreste (Alves family) and northern Atlantic forest (Dantas family) of Pernambuco. NBSS = Number of body systems influenced by the cited species; NP = Number of properties attributed to the cited species; $\mathrm{RI}=$ Relative importance

\begin{tabular}{|c|c|c|c|c|c|c|c|}
\hline Specie & $\begin{array}{l}\text { Common } \\
\text { name }\end{array}$ & $\begin{array}{l}\text { Number of } \\
\text { informants }\end{array}$ & $\begin{array}{l}\text { Number of body } \\
\text { systems treated }\end{array}$ & $\begin{array}{l}\text { Number of } \\
\text { properties } \\
\text { attributed }\end{array}$ & NBSS & $\mathbf{N P}$ & $\mathbf{R I}$ \\
\hline $\begin{array}{l}\text { Cymbopogon citratus } \\
\text { D.C Stapf }\end{array}$ & $\begin{array}{l}\text { Capim santo } \\
\text { (Lemon grass) }\end{array}$ & 15 & 3 & 7 & 0.75 & 0.88 & 1.63 \\
\hline Mentha sp. & $\begin{array}{l}\text { Hortelã da } \\
\text { folha miúda } \\
\text { (Small leaf } \\
\text { mint) }\end{array}$ & 11 & 3 & 7 & 0.75 & 0.88 & 1.63 \\
\hline Punica granata L. & $\begin{array}{l}\text { Romã } \\
\text { (Pomegranate) }\end{array}$ & 10 & 4 & 5 & 1.00 & 0.63 & 1.63 \\
\hline $\begin{array}{l}\text { Lippia alba (Mill) } \\
\text { Brow }\end{array}$ & Erva cidreira & 10 & 4 & 8 & 1.00 & 1.00 & 2.00 \\
\hline
\end{tabular}




\begin{tabular}{|c|c|c|c|c|c|c|c|}
\hline \\
\hline $\begin{array}{l}\text { Myracrodruon } \\
\text { urundeuva (Engl.) Fr. } \\
\text { Allemão }\end{array}$ & Aroeira & 10 & 4 & 6 & 1.00 & 0.75 & 1.75 \\
\hline $\begin{array}{l}\text { Aloe vera (L.) Burm. } \\
\text { f. }\end{array}$ & Babosa & 9 & 3 & 4 & 0.75 & 0.5 & 1.25 \\
\hline Eucalyptus sp. & $\begin{array}{l}\text { Eucalipto da } \\
\text { folha fina } \\
\text { (Narrow leaf } \\
\text { eucalyptus) }\end{array}$ & 7 & 3 & 3 & 0.75 & 0.38 & 1.13 \\
\hline $\begin{array}{l}\text { Jatropha } \\
\text { gossypiifolian L. }\end{array}$ & Pinhão roxo & 7 & 2 & 2 & 0.5 & 0.25 & 0.75 \\
\hline $\begin{array}{l}\text { Mesosphaerum } \\
\text { pectinatum (L.) } \\
\text { Kuntze }\end{array}$ & Samba caité & 7 & 4 & 6 & 1.00 & 0.75 & 1.75 \\
\hline $\begin{array}{l}\text { Acanthospermum } \\
\text { hispidum D.C }\end{array}$ & $\begin{array}{l}\text { Espinho de } \\
\text { cigano }\end{array}$ & 7 & 1 & 3 & 0.25 & 0.38 & 0.63 \\
\hline
\end{tabular}

The parts of the plants most cited by the informants (Graph No. 1) were the leaves $(31.16 \%)$, followed by the stems $(19.60 \%)$ and fruits $(18.09 \%)$. Other parts also used were the seeds (10.05\%), flowers $(8.54 \%)$, roots $(8.03 \%)$, pseudofruits $(1.01 \%)$, and infructescences $(1.01 \%)$. The use of the whole plant was infrequent $(2.51 \%)$.

Graph No. 1

Parts of the plants cited for various uses by Roma informants living in the Agreste (Alves family) and northern Atlantic forest (Dantas family) of Pernambuco

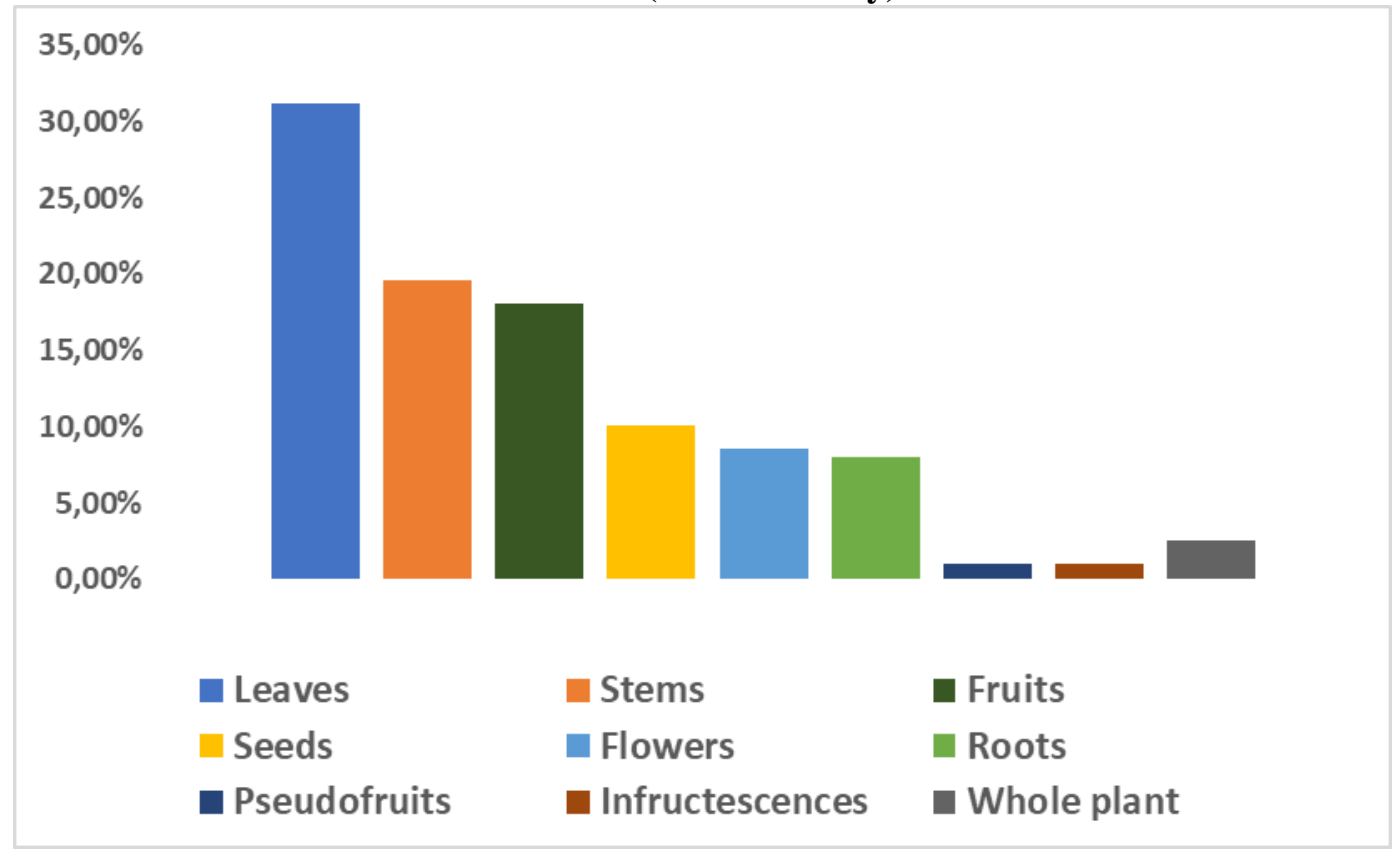

Approximately half of the mentioned species (54\%) were native to Brazil, with the rest being exotic, cultivated $(25 \%)$ or naturalized $(21 \%)$.

Most of the plants mentioned (52\%) had medicinal uses. They were indicated for the treatment of diseases related to different body systems, but also for food (18\%), cosmetic (7\%), ritualistic (7\%), condiment $(5 \%)$, veterinary $(5 \%)$, technological $(4 \%)$ purposes, and $2 \%$ were indicated for other uses, such as insecticides, abortive agents, or appetite stimulants (Graph No. 2). 
Graph No. 2

Categories of use cited by Roma informants living in the Agreste (Alves family) and northern Atlantic forest (Dantas family) of Pernambuco

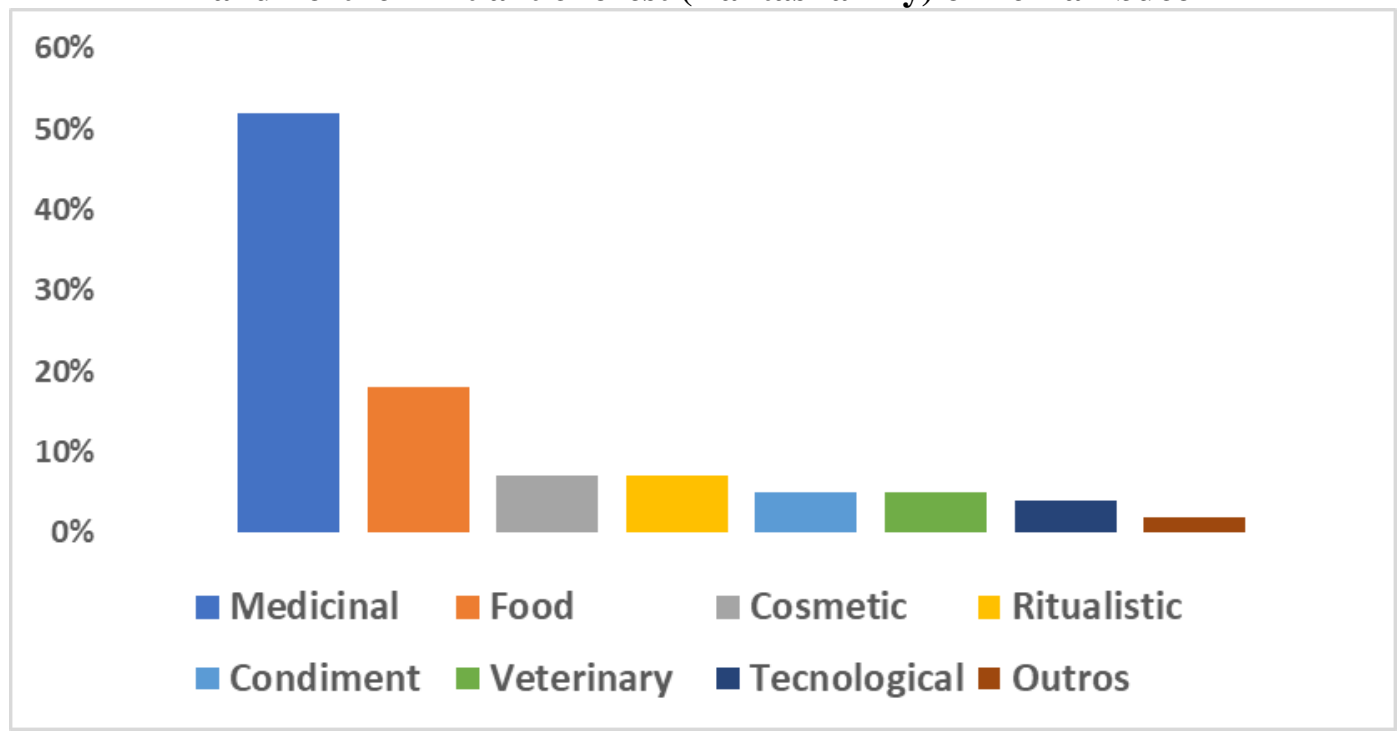

\section{DISCUSSION}

Greatest knowledge about the taxonomic diversity was observed among the Alves family; however, there was a similarity of 0.66 between the informants in terms of the plant families mentioned, which were also the most frequent in the lists of plant families used by non-Roma communities in northeastern Brazil. The preference for leaves and stems is probably due to their easy collection and because they are a permanent resource, unlike flowers, fruits and seeds, which are mostly seasonal.

The higher percentage of native species used by Roma people (54\%) differs from what has been described for non-Roma communities living in the same regions (Sousa et al., 2018; Brito et al., 2015; Lima, 2015), but the distribution in relation to the categories of use was very similar, with predominance of medicinal use (Lopes \& Lobão, 2013; Andrade et al., 2018; Oliveira et al., 2018). The outstanding position of the species L. alba in terms of relative importance, use value and reliability index corroborates what is found in the scientific literature on the use of medicinal plants by non-Roma populations in northeastern Brazil (Andrade et al., 2018; Oliveira et al., 2018).

Men showed a slightly greater ethnobotanical knowledge compared to women. This is in contrast with surveys conducted with non-Roma communities in the same region (Albuquerque \& Andrade, 2002; Rodrigues \& Andrade, 2014; Lima, 2015). Considering that practically the same number of men and women were interviewed, this finding can be explained by the dominance of men peculiar of Roma culture. The greater ethnobotanical knowledge of male individuals aged 50 to 60 years can also have a cultural reason, since the ages of two of the three family heads interviewed were within this range. In the case of women, ethnobotanical knowledge is better distributed among age groups.

Factors related to the Roma culture, such as the language, the importance of kinship relations, the transmission of oral knowledge, nomadic or seminomadic behavior, and the trader tradition, may be responsible for the similarity observed between these families, as their members do not know each other, nor have direct contact.

A comparison between the set of plants used by the Dantas and Alves families with the lists of useful species found in ethnobotanical surveys of non-Roma communities living in the northern Atlantic forest (Silva \& Andrade, 2005; Rodrigues \& Andrade, 2014) and the Agreste (Albuquerque \& Andrade, 2002; Teixeira \& Melo, 2006; Carvalho et al., 2013) of Pernambuco revealed 76 and 95 shared species, respectively, which corresponds to $75-70 \%$ of the total cited. Besides this similarity in the lists of 
species, there was also a significant approximation in relation to their respective uses. However, some uses mentioned by the participants were original, as in the case of $H$. indicum and A. congestiflora, indicated for the treatment of Newcastle disease in poultry (Lobo et al., 2020).

The results showed that there was no relevant difference between the ethnobotanical knowledge of Roma families and non-Roma populations living in the same region. This similarity, also observed in daily clothing, must be related to the tendency of Roma families to adopt some customs of local nonRoma communities. This could be considered as a kind of mimicry that allows them to live with less discrimination, yet without losing their cultural identity.

\section{CONCLUSION}

The interaction with other peoples, the practice of informal commerce, the discrimination suffered over the centuries, and the need to survive have led the Roma people to develop what could be interpreted as cultural mimicry, adapting to each region they pass through and inhabit. This adaptation can be observed also in the use of the flora, for the communities approached in the present study demonstrated a very similar knowledge to that of the non-Roma communities with which they have come into contact. Measuring this adaptation or the influence of this culture in the formation of the Brazilian population is a difficult and multidisciplinary task, which requires greater commitment from the scientific community.

\section{REFERENCES}

Albuquerque UP, Andrade LHC. 2002. Conhecimento botânico tradicional e conservação em uma área de caatinga no estado de Pernambuco, Nordeste do Brasil. Acta Bot Bras 16: 273 - 285. https://doi.org/10.1590/s0102-33062002000300004

Albuquerque UP, Silva VA, Cabral MC, Alencar NL, Andrade LHC. 2008. Comparisons between the use of medicinal plants in indigenous and rural Caatinga (dryland) communities in NE Brazil. Bol Latinoam Caribe Plant Med Aromat 7: 156 - 170.

Albuquerque UP de, Lucena RFP de, Cunha LVFC da. 2010. Métodos e Técnicas na Pesquisa Etnobiológica e Etnoecológica. Ed. Comunigraf/ NUPEEA, Recife, Brasil.

Andrade AMF de, Alves CAB, Souza RS, Silva S da. 2018. Inventário etnobotânico e uso das espécies madeireiras e não madeireiras na comunidade de Ouricuri, Pilões-PB, Nordeste do Brasil. Rev Equador 8: 399 - 421.

Andrade Júnior L. 2013. Os ciganos e os processos de exclusão. Rev Bras Hist 33: 95 - 112. https://doi.org/10.1590/s0102-01882013000200006

Bennett BC; Prance GT. 2000. Introduced plants in the indigenous pharmacopoeia of Northen South America. Econ Bot 54: 90 - 102. https://doi.org/10.1007/bf02866603

Brito MFM, Lucena RFP, Cruz DD. 2015. Conhecimento etnobotânico local sobre plantas medicinais: uma avaliação de índices quantitativos. Interciencia 40: 156 - 164.

Carvalho JSB, Martins JDL, Mendonça MCS, Lima LD. 2013. Uso popular das plantas medicinais na comunidade da Várzea, Garanhuns - PE. Rev Biol Ciênc Terra 13: 58 - 65.

Coelho FA. 1892. Os Ciganos de Portugal. Imprensa Nacional, Lisboa, Portugal.

Derlon P. 1979. A medicina secreta dos ciganos. Difel/Difusão Editorial S/A, Algés, Portugal.

Gresham D. 2001. Origins and divergence of the Roma (gypsies). Am J Hum Gen 69: 1314 - 1331.

Flora do Brasil 2020 em construção. Jardim Botânico do Rio de Janeiro. http://floradobrasil.jbrj.gov.br

Lima VHM. 2015. Uso e conhecimento de plantas medicinais utilizadas pelas mulheres da comunidade Mendes, Limoeiro, Pernambuco, Brasil. Revista Ouricuri 5: 168 - 182.

Lobo RAAM, Lobo ACBNM, Oliveira AFM, Andrade LHC. 2020. Ethnomedicinal plants for veterinary use in gypsy communities of the northeast of Brazil. Bol Latinoam Caribe Plant Med Aromat 19: 179 - 187.

Lopes LCM, Lobão AQ. 2013. Etnobotânica em uma comunidade de pescadores artesanais no litoral norte do Espírito Santo, Brasil. Bol Museu Biol Mello Leitão 32: 29 - 52.

Martins ESS, Costa JC. 2016. Importância relativa das plantas medicinais comercializadas nas feiras dos municípios de Paulo Afonso - Bahia, Delmiro Gouveia - Alagoas e Petrolândia - Pernambuco, Brasil. Opará: Etnicidades, Movimentos Sociais e Educação 4: 63 - 80.

Boletín Latinoamericano y del Caribe de Plantas Medicinales y Aromáticas / 546 
Mendizabal I, Lao O, Marigorta UM, Wollstein A, Gusmão L, Ferak V, Ioana M, Jordanova A, Kaneva R, Kouvatsi A, Kučinskas V, Makukh H, Metspalu A, Netea MG, Pablo R, Pamjav H, Radojkovic D, Rolleston SJH, Kayser M. 2012. Reconstructing the population history of European Romani from genome-wide data. Curr Biol 22: 2342 - 2349. https://doi.org/10.1016/j.cub.2012.10.039

SGM - MME. (Ministério de Minas e Energia). 2005. Secretaria de Geologia, Mineração e Transformação Mineral. Projeto cadastro de fontes de abastecimento por àgua subterrânea. Brasília, Brasil.

Moonen F. 2011. Anticiganismo: os ciganos na Europa e no Brasil. Ed. Digital. Recife, Brasil.

Oliveira DM de, Santos LAS, Gomes LJ. 2018. Uso da flora em assentamento agroextrativista do litoral de Sergipe, Brasil. Guaju 4: 163 - 183. https://doi.org/10.5380/guaju.v4i1.56407

Prance GT, Balée W, Boom BM, Carneiro RL. 1987. Quantitative ethnobotany and the case for conservation in ammonia. Conserv Biol 4: 296 - 310. https://doi.org/10.1111/j.1523-1739.1987.tb00050.x

Ramanush N. 2011. Cultura cigana, nossa História por nós. Ensaio 1: 9 - 13.

Rodrigues AP, Andrade LHC. 2014. Levantamento etnobotânico das plantas medicinais utilizadas pela comunidade de Inhamã, Pernambuco, Nordeste do Brasil. Rev Bras Plant Med 16: 721 - 730. https://doi.org/10.1590/1983-084x/08_159

Seraj S, Jahan FI, Chowdhury AR, Monjur-Ekhuda M, Khan MS, Aporna SA, Jahan R, Samarrai W, Islam F, Khatun Z, Rahmatullah M. 2013. Tribal formulations for treatment of pain: a study of the bede community traditional medicinal practitioners of porabari village in Dhaka district, Bangladesh. Afric J Trad Complement Alt Med 10: 26 - 34. https://doi.org/10.4314/ajtcam.v10i1.5

Silva AJR, Andrade LHC. 2005. Etnobotânica nordestina: estudo comparativo da relação entre comunidades e vegetação na Zona do Litoral - Mata do Estado de Pernambuco, Brasil. Acta Bot Bras 19: 45 - 60. https://doi.org/10.1590/s0102-33062005000100006

Simões SRCF. 2014. Vida cigana: aspectos que configuram as atuais dinâmicas das mudanças dos ciganos brasileiros. Thesis, Universidade do Sul de Santa Catarina, Palhoça, SC, Brasil.

Sousa VFO, Santos GL dos, Rodrigues MHBS, Barroso RF, Leitão ETC, Santos JJF dos. 2018. Levantamento etnobotânico da Universidade Federal de Campina Grande, Campus Pombal. Acta Biol Catarinense 5: 46 - 55. https://doi.org/10.21726/abc.v5i1.516

Teixeira RC. 2008. História dos Ciganos no Brasil. Núcleo de Estudos Ciganos. Recife, Brasil.

Teixeira AS, Melo JIM de. 2006. Plantas medicinais utilizadas no município de Jupi, Pernambuco, Brasil. Iheringia (Série Botanica) 61: 5 - 11.

Wulf C. 2016. Aprendizagem cultural e mimese: jogos, rituais e gestos. Rev Bras Educ 21: 553 - 568. https://doi.org/10.1590/s1413-24782016216629 